\title{
Pueblos indígenas y cambio climático: el caso de la Amazonía colombiana
}

Peuples indigènes et changements climatiques

Indigenous people and climatic change

\section{Juan Álvaro Echeverri}

\section{(2) OpenEdition}

\section{Journals}

Edición electrónica

URL: http://journals.openedition.org/bifea/2774

DOI: $10.4000 /$ bifea. 2774

ISSN: 2076-5827

\section{Editor}

Institut Français d'Études Andines

Edición impresa

Fecha de publicación: 1 abril 2009

Paginación: 13-28

ISSN: 0303-7495

Referencia electrónica

Juan Álvaro Echeverri, «Pueblos indígenas y cambio climático: el caso de la Amazonía colombiana », Bulletin de l'Institut français d'études andines [En línea], 38 (1) | 2009, Publicado el 01 octubre 2009, consultado el 17 noviembre 2020. URL : http://journals.openedition.org/bifea/2774 ; DOI : https:// doi.org/10.4000/bifea.2774

\section{(c) $98 \%$}

Les contenus du Bulletin de l'Institut français d'études andines sont mis à disposition selon les termes de la licence Creative Commons Attribution - Pas d'Utilisation Commerciale - Pas de Modification 4.0 International. 


\title{
Pueblos indígenas y cambio climático: el caso de la Amazonía colombiana
}

\author{
Juan Álvaro Echeverri*
}

\begin{abstract}
Resumen
Este artículo analiza las percepciones de los pueblos indígenas de la Amazonía colombiana acerca de los cambios climáticos ocurridos durante los últimos 10 años y los efectos directos sobre sus modos de subsistencia. En la primera sección, se presentan cambios en temperatura y estacionalidad y se contrastan con el conocimiento indígena sobre el calendario ecológico. En la siguiente sección, se exponen los principales efectos directos de esos cambios sobre los modos de subsistencia indígena, principalmente sobre la horticultura, la disponibilidad de recursos acuáticos y la salud. Se realiza también una discriminación de los efectos según franjas de género y edad, y según el grado de autonomía territorial de los grupos indígenas. Con estos insumos se discuten las estrategias y adaptaciones de los indígenas para acomodarse a estos cambios y se subraya, finalmente, que el impacto del cambio climático global en esta región solo puede ser entendido y evaluado en conjunción con todos los otros cambios sociales que afectan la región.
\end{abstract}

Palabras clave: cambio climático, Amazonía colombiana, calendario ecológico, pueblos indígenas

\section{Peuples indigènes et changements climatiques}

\section{Résumé}

Cet article analyse la perception qu'ont les peuples indigènes de l'Amazonie colombienne des changements climatiques intervenus durant les dix dernières années et, en particuliers, des effets directs de ces changements sur leur mode de subsistance. La première section présente les changements de température et de saisons que l'on compare avec la connaissance indigène du calendrier écologique. La section suivante présente les principaux effets directs de ces changements sur les modes de subsistance indigène, notamment sur l'horticulture, la disponibilité des ressources en eau et la santé. II

* Instituto Amazónico de Investigaciones (IMANI) de la Universidad Nacional de Colombia (Sede Amazonía).

E-mail: juanalvaroe@gmail.com 
apparaît que les effets ne sont pas les mêmes suivant les tranches d'âge et le genre, et suivant le degré d'autonomie territoriale des groupes indigènes. Partant de ces données, l'auteur discute les stratégies d'adaptation des indigènes face à ces changements, pour souligner finalement le fait que l'impact du changement climatique global dans cette région ne peut être compris et évalué en écartant les autres changements sociaux qui affectent la région.

Mot clés : changement climatique, Amazonie colombienne, calendrier écologique, peuples indigènes

\title{
Indigenous people and climatic change
}

\begin{abstract}
This article discusses the perceptions of indigenous peoples of the Colombian Amazon of climate changes during the last ten years, and the direct effects of those changes on indigenous livelihood. In the first section, changes in temperature and seasonality are presented and contrasted with indigenous traditional knowledge on the seasonal ecological calendar. In the next section, the main direct effects of these changes on indigenous livelihood are presented, mainly on horticulture, availability of fish and human health. In addition, these effects are differentiated according to gender and age groups, and according to the degree of territorial autonomy of different indigenous groups. With this information, the strategies and adaptations of indigenous peoples to cope with these changes are discussed. We show, finally, that the impact of global climate change in this region should be assessed together with other social changes affecting it.
\end{abstract}

Key words: climate change, Colombian Amazon, ecological calendar, indigenous people

\section{INTRODUCCIÓN}

El cambio climático es un tema que ha acaparado la atención mundial en los últimos años. Sin embargo, gran parte de esta atención ha estado enfocada en los aspectos físicos y naturales del cambio climático, o en el de las responsabilidades políticas y económicas de los países y empresas que contribuyen, en mayor medida, a las emisiones de gas carbónico en la atmósfera terrestre. Los aspectos sociales, en cambio, han tenido mucho menos relieve, aunque es evidente que los pobres del mundo y los pueblos indígenas están siendo y continuarán siendo los más afectados por los efectos, aún no enteramente predecibles, de las alteraciones del clima —ver, por ejemplo, las escasas menciones a estos aspectos en el informe del Panel Intergubernamental sobre Cambio Climático (IPCC, 2007)—. En este artículo, presento una primera aproximación a la percepción indígena de cambios climáticos en los últimos años, los impactos directos y potenciales de esos cambios en el modo de vida indígena, la interpretación nativa de esos eventos y las adaptaciones a los cambios percibidos.

Me baso en información primaria obtenida con diferentes grupos indígenas del sur y oriente de la Amazonía colombiana, con mayor énfasis en los grupos del interfluvio CaquetáPutumayo (uitoto, muinane, nonuya) y del Trapecio amazónico colombiano (ticuna y cocama). La información presentada aquí se apoya en tres fuentes. En primer lugar, una serie de entrevistas semiestructuradas con 25 hombres y mujeres indígenas, mayores y jóvenes, 
de esas dos regiones, entre abril y mayo de 20051. Este sondeo preliminar tuvo por objeto tener una primera evaluación de la percepción y efectos de cambios en el régimen de estacionalidad, conectados con cambios climáticos globales, en esta parte de la Amazonía. Esta información se contrastó y evaluó de dos maneras. Por una parte se obtuvieron los registros mensuales de temperatura, precipitación y niveles fluviales en 10 estaciones meteorológicas del Instituto de Hidrología, Meteorología y Estudios Ambientales (IDEAM, Colombia) para todas las fechas disponibles (desde 1970 hasta 2007)2. Sobre la base de esta información sobre los parámetros climáticos básicos en diferentes puntos de la región en las últimas décadas, hago referencias a eventos o comportamientos del clima, y los comparo con las percepciones obtenidas en las encuestas, en diferentes puntos del texto. Por otra parte, me apoyé en mi conocimiento directo y experiencia de trabajo, sobre todo con los grupos del interfluvio Caquetá-Putumayo; las referencias cosmológicas sobre el calendario ecológico y las normas de comportamiento y prevención asociadas a las estaciones que introduzco en el texto son características de los grupos del interfluvio Caquetá-Putumayo. Las siguientes observaciones hacen referencia, principalmente, a la percepción de eventos de cambio climático en el Trapecio amazónico y en el interfluvio Caquetá-Putumayo.

Una percepción generalizada entre estos grupos es que, desde la década de 1990, se han hecho evidentes alteraciones en el clima (particularmente en la estacionalidad) y estos cambios se han vuelto ostensibles desde el año 2005. Los grupos indígenas amazónicos, en general, son agudos observadores de los ritmos naturales y han acumulado un amplio y sofisticado conocimiento de los ciclos estacionales. Sus sistemas de vida (horticultura de roza y quema, pesca, cacería y recolección de frutos y materiales silvestres) están muy ligados a la sucesión de estaciones bien establecidas. Los indígenas son conscientes de índices ecológicos complejos que ordenan la interrelación de los ciclos del agua, los vientos y la temperatura, y de sus efectos en los ciclos reproductivos de los peces, la fauna terrestre y los frutales silvestres, y en la regulación de las actividades rituales y productivas de la gente. Aunque es normal que la sucesión estacional presente variaciones interanuales, según varios indígenas entrevistados en diferentes áreas, los signos e índices naturales que ahora perciben son «alarmantes»: las estaciones están ocurriendo fuera del tiempo, los pulsos de inundación y descenso de los ríos están desincronizados con la maduración de los frutos silvestres y el calor está aumentando, entre otros acontecimientos. Más que el impacto del incremento de la temperatura ambiente, son los cambios en la precipitación y la estacionalidad los que tienen mayor impacto en las actividades de subsistencia, principalmente en la horticultura, la reproducción de los peces y en la salud humana, como explicaremos más adelante 3 .

1 Se hicieron entrevistas con personas de las siguientes comunidades: en el río Amazonas, Leticia, Puerto Nariño, El Castañal, San Sebastián, Kilómetro 11 y Ronda; en el río Caquetá, Araracuara y Peña Roja; y en el río Putumayo y afluentes, La Chorrrera y Cordillera.

2 Estaciones meteorológicas: río Amazonas: Aeropuerto Vásquez Cobo, Leticia $\left(4^{\circ} 12^{\prime} \mathrm{S}, 69^{\circ} 57^{\prime} \mathrm{W}-1970-2007\right)$, Bocatoma $\left(04^{\circ} 11^{\prime} \mathrm{S}, 69^{\circ} 57^{\prime} \mathrm{W}\right.$ - 1990-2007), Yahuarcaca $\left(4^{\circ} 11^{\prime} \mathrm{S}, 69^{\circ} 57^{\prime} \mathrm{W}\right.$ - 2000-2007); río Caquetá: Araracuara $\left(0^{\circ} 37^{\prime} \mathrm{S}, 72^{\circ} 24^{\prime} \mathrm{W}\right.$ - 1979-2007), Las Mercedes $\left(0^{\circ} 32^{\prime} \mathrm{S}, 72^{\circ} 10^{\prime} \mathrm{W}\right.$ - 1987-2005), La Pedrera (01¹8' S, 69³7’ W - 1984-2007); río Putumayo: Puerto Leguízamo $\left(00^{\circ} 19^{\prime} \mathrm{S}, 74^{\circ} 46^{\prime} \mathrm{W}\right.$ - 1976-2007), Puerto Tolosa $\left(01^{\circ} 02^{\prime} \mathrm{S}, 74^{\circ} 10^{\prime} \mathrm{W}\right.$ - 1986-2007), Estrecho-Marandúa $\left(02^{\circ} 30^{\prime} \mathrm{S}, 72^{\circ} 40^{\prime} \mathrm{W}-2000-2007\right)$ y Puerto Arica $\left(02^{\circ} 08^{\prime} \mathrm{S}, 71^{\circ} 44^{\prime} \mathrm{W}-1986-2007\right)$.

3 El régimen de precipitación de la cuenca amazónica depende de la temperatura superficial del mar (Salick \& Byg, 2007) y de la interacción mar-bosque (Bunyard, 2008). Los bosques tropicales ya han sido profundamente afectados por cambios climáticos causados por la oscilación meridional de El Niño y La Niña. Estos efectos sobre la cuenca amazónica son, además, exacerbados por la tala de árboles y la fragmentación del bosque (Bunyard, 2008). 
Esta tendencia general, sin embargo, tiene efectos diferenciados según la gama de la variación natural, cultural y social a lo largo de la región. La Amazonía colombiana está situada en la porción noroccidental de la cuenca amazónica, es una región de alta diversidad cultural y natural, y la cobertura vegetal está mucho mejor conservada que en otras partes de la Amazonía. Al estar situada en la zona de interconfluencia tropical, el clima de la Amazonía colombiana varía a lo largo del eje norte-sur, con dos regímenes distintos de precipitación. Las condiciones sociales y culturales también son variables. La Amazonía colombiana alberga 52 grupos étnicos pertenecientes a 13 familias lingüísticas, más 10 lenguas aisladas. Además, hay un claro contraste entre los grupos indígenas de la parte occidental de la Amazonía colombiana —-donde la colonización, el conflicto armado, los cultivos ilegales y la deforestación han tenido mayor impacto- y los grupos de la parte oriental, que conservan mayor autonomía territorial y cultural.

Los indígenas han venido percibiendo y sufriendo efectos directos del cambio climático global, aunados con otros factores que también afectan los modos de vida indígena. Los impactos negativos del cambio climático se combinan con la explotación de los recursos naturales (madera, oro, coca, pesca comercial), el conflicto armado, la presencia creciente de la educación escolarizada y los medios masivos y las relaciones crecientes de los indígenas con instituciones gubernamentales y no gubernamentales. La interpretación indígena de estas tendencias complejas se enmarca en sus tradiciones culturales. Para los grupos más tradicionales, la alteración de los ciclos naturales y sus efectos en su subsistencia y salud se interpretan en un marco moral y ético, con un sentido de responsabilidad compartida. Los indígenas del Medio Caquetá señalan que si bien puede ser cierto que la «gente blanca» ha causado daño al planeta, lo que está en la raíz del mal funcionamiento de la naturaleza son sus propias fallas en observar las normas y los principios de comportamiento entregados por el Creador. Es la sociedad, no la naturaleza, lo que orienta la interpretación indígena del cambio climático.

En la siguiente sección, presento las principales percepciones y constataciones de cambios recientes en el clima, sobre todo en los aspectos de temperatura y estacionalidad. En la subsiguiente sección, expongo los principales efectos directos de esos cambios en los modos de subsistencia indígena, según la percepción de la gente. Con estas informaciones, discuto las estrategias y adaptaciones de los indígenas para lidiar con estos cambios, y subrayo, finalmente, que el impacto del cambio climático global en esta región solo puede ser entendido y evaluado en conjunto con todos los otros cambios sociales que afectan la región.

\section{TEMPERATURA Y ESTACIONALIDAD}

Un primer efecto del cambio climático es el incremento de la temperatura ambiente en lo que va de este siglo. Un indígena del medio río Caquetá lo ilustra con una observación simple: antes (hace más de 10 años), a partir de las 6 de la tarde, el calor del día se mantenía hasta las 9 ó 10 pm, y luego el ambiente se refrescaba; hoy en día, en cambio, el calor del día se continúa sintiendo incluso después de medianoche. El incremento de temperatura afecta sobre todo el trabajo hortícola. Las chacras o parcelas hortícolas son áreas abiertas donde el calor se siente fuertemente. Hoy en día, se ha vuelto imposible estar de pie descalzo al mediodía sobre el suelo de la chacra. La gente ahora tiene que abandonar temprano sus chacras, acortando el tiempo de trabajo hortícola. 
Esta percepción se correlaciona bien con datos climatológicos locales4. Las temperaturas en la Amazonía tienen un ciclo anual de variación, con índices más bajos hacia la mitad del año (invierno del hemisferio austral). Para Puerto Leguízamo (río Putumayo), los datos de temperatura del periodo 2000-2007 muestran un incremento promedio de $0,5^{\circ} \mathrm{C}$ con respecto a los promedios históricos (1976-2007). Para Leticia (río Amazonas), el promedio del periodo 2000-2007 no muestra incrementos significativos, con respecto al histórico (1970-2007), a excepción de los meses finales del año (usualmente los más cálidos). En cambio, en Puerto Leguízamo los datos de temperatura para el año 2005 registran un incremento de casi $1^{\circ} \mathrm{C}$, con respecto a los promedios históricos, en todos los meses del año (fig. 1).

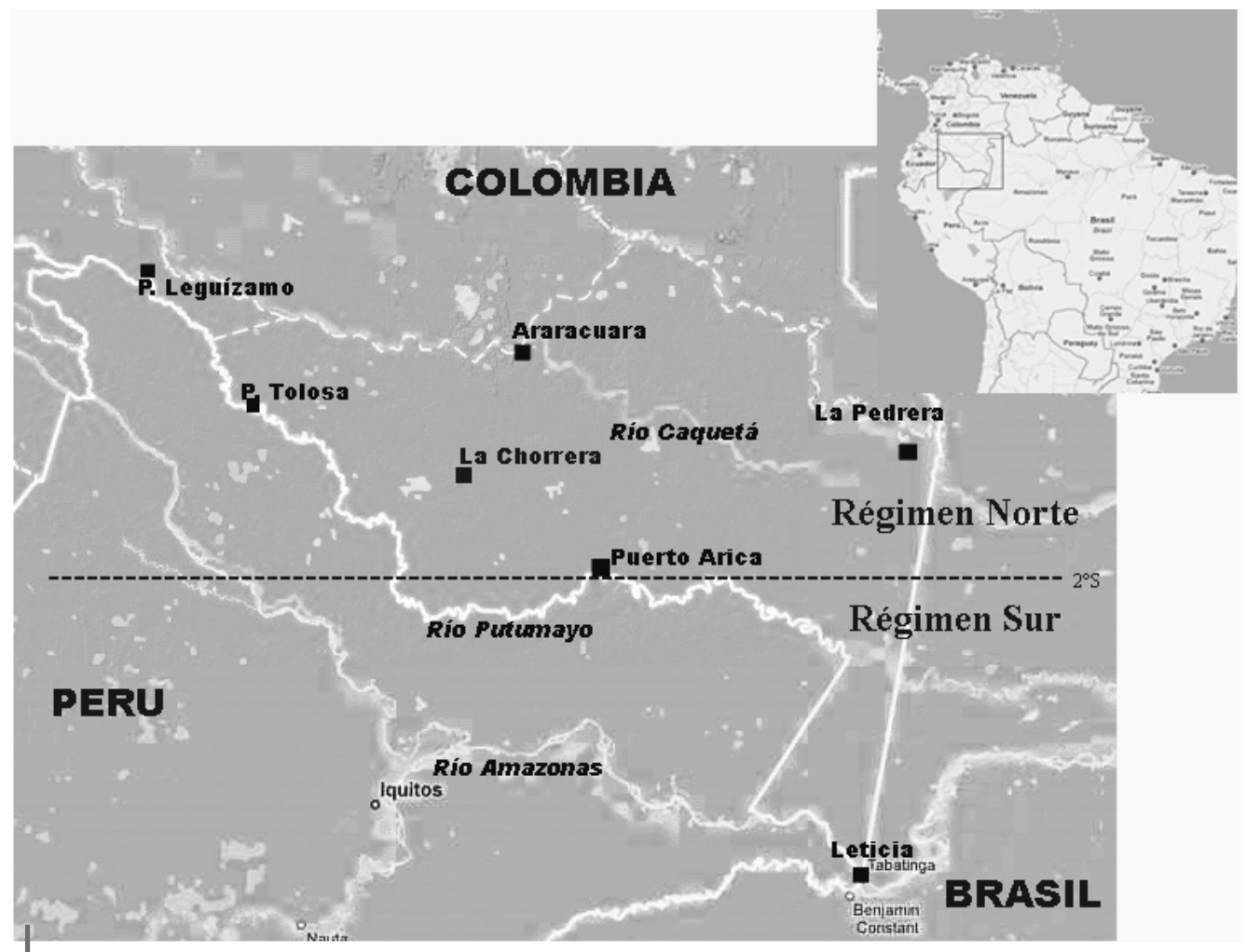

Figura 1 - Amazonía colombiana: regímenes estacionales norte y sur

Cartografía base: Google Maps <http://maps.google.com/maps?f=q\&source=s_q\&hl=en\&geocode $=\& q=$ colombia\&s $\|=37.0625,-95.677068 \& s s p n=41.360684,64.160156 \& \mathrm{ie}=\mathrm{UTF} 8 \&\|=-1.669686,-71.553955 \& \mathrm{spn}=6.541519,12.194-$ $824 \& \mathrm{t}=\mathrm{p} \& \mathrm{z}=7>$

Resaltamos la existencia de dos regímenes estacionales, al nortey al sur del paralelo $2^{\circ} \mathrm{S}$ (fig. 1 ). El régimen norte es común para la mayor parte de la Amazonía colombiana y regiones vecinas del Amazonas venezolano y del norte del Brasil; el régimen sur se aplica para Colombia y es compartido por la mayor parte del Amazonas peruano y brasileño.

4 Datos meteorológicos de las estaciones Aeropuerto Vásquez Cobo, Leticia (412'S, 6957'W - 1970-2007) y Puerto Leguízamo $\left(00^{\circ} 19^{\prime} \mathrm{S}, 74^{\circ} 46^{\prime} \mathrm{W}\right.$ - 1976-2007). 
Estos regímenes estacionales diferenciados propician que las alteraciones climáticas no sean uniformes para todas las áreas. La figura 2 muestra los datos históricos para los valores promedios de precipitación y niveles del río en Araracuara (régimen Norte) y en Leticia (régimen Sur).
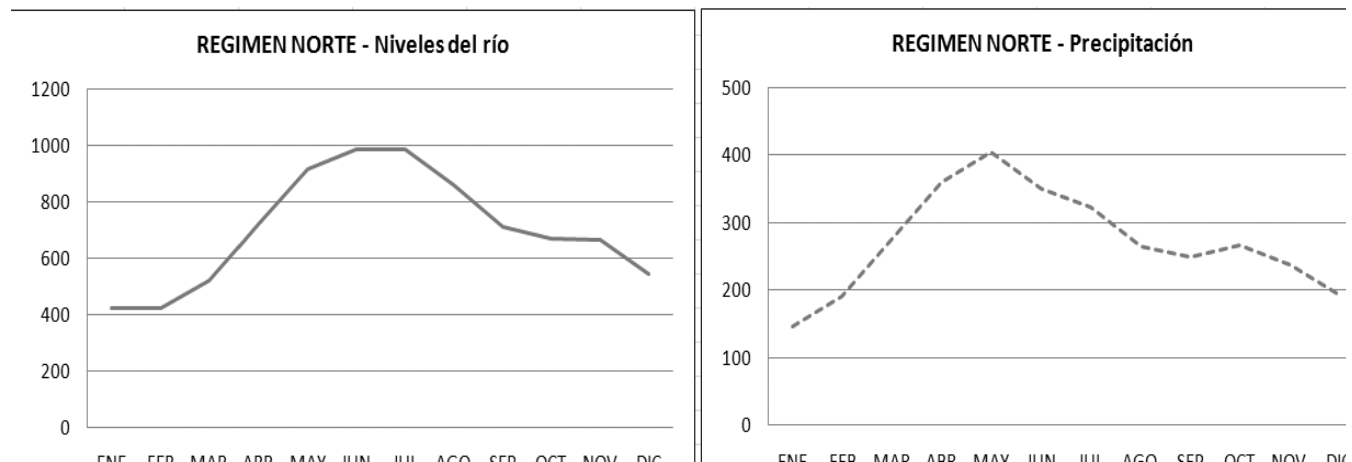

ENE FEB MAR ABR MAY JUN JUL AGO SEP OCT NOV DIC ENE FEB MAR ABR MAY JUN JUL AGO SEP OCT NOV DII

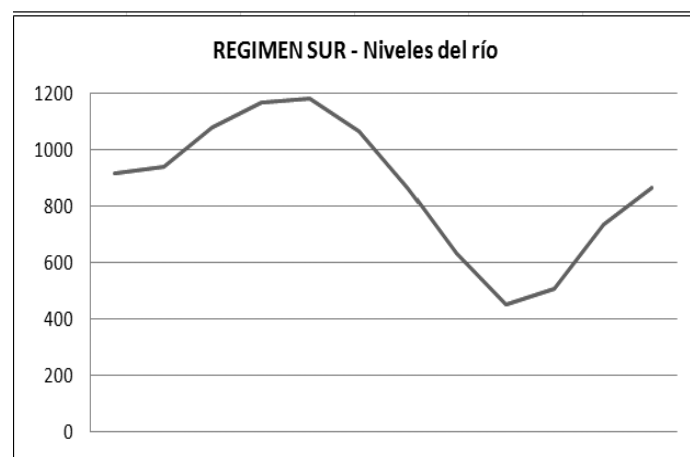

ENE FEB MAR ABR MAY JUN JUL AGO SEP OCT NOV DIC

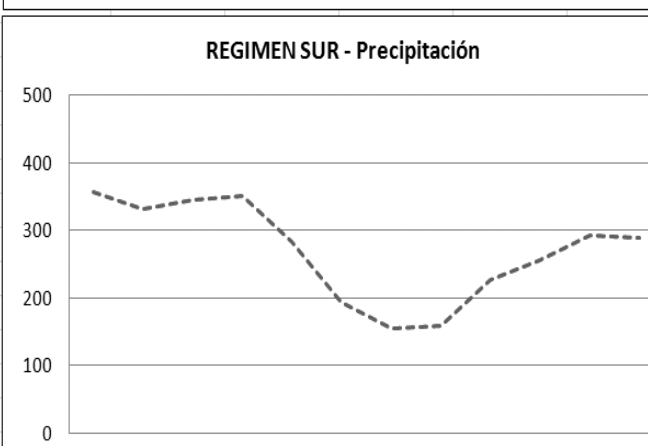

ENE FEB MAR ABR MAY JUN JUL AGO SEP OCT NOV DI

Figura 2 - Regímenes estacionales Norte y Sur de la Amazonía colombiana

Elaborado a partir de datos meteorológicos de las estaciones: Araracuara $\left(0^{\circ} 37^{\prime} \mathrm{S}, 72^{\circ} 24^{\prime} \mathrm{W}\right)$, valores medios para el periodo 1979-2007, para el régimen norte; y Aeropuerto Vásquez Cobo (Leticia) $\left(4^{\circ} 12^{\prime} \mathrm{S}, 69^{\circ} 57^{\prime} \mathrm{W}\right)$, precipitación media para el periodo 1970-2007, y Yahuarcaca $\left(4^{\circ} 11^{\prime} \mathrm{S}, 69^{\circ} 57^{\prime} \mathrm{W}\right)$, niveles medios del río para el periodo $2000-2007$, para el régimen sur (Colombia, IDEAM, 2008)

La sucesión anual de estaciones es de la mayor importancia para la gente indígena. Este ritmo ordena los tiempos del ciclo hortícola y de las prácticas rituales que ayudan a prevenir enfermedades y a promover bienestar humano, y es crucial para la reproducción de la vida silvestre. Los indígenas del interfluvio Caquetá-Putumayo conciben el ciclo anual como establecido por el «Padre Creador» desde el principio de los tiempos y conocen bien los fundamentos ecológicos y éticos de este ciclo y de los efectos adversos de su alteración. Para ilustrar el sentido que dan los indígenas a la sucesión anual, incluyo una versión resumida del calendario ecológico como se supone que tradicionalmente debe ocurrir, narrada por indígenas de la etnia nonuya, que viven sobre el medio río Caquetá (casi sobre la línea ecuatorial), correspondiendo al régimen estacional Norte. La figura 3 ilustra, de manera sintética, la narración. 


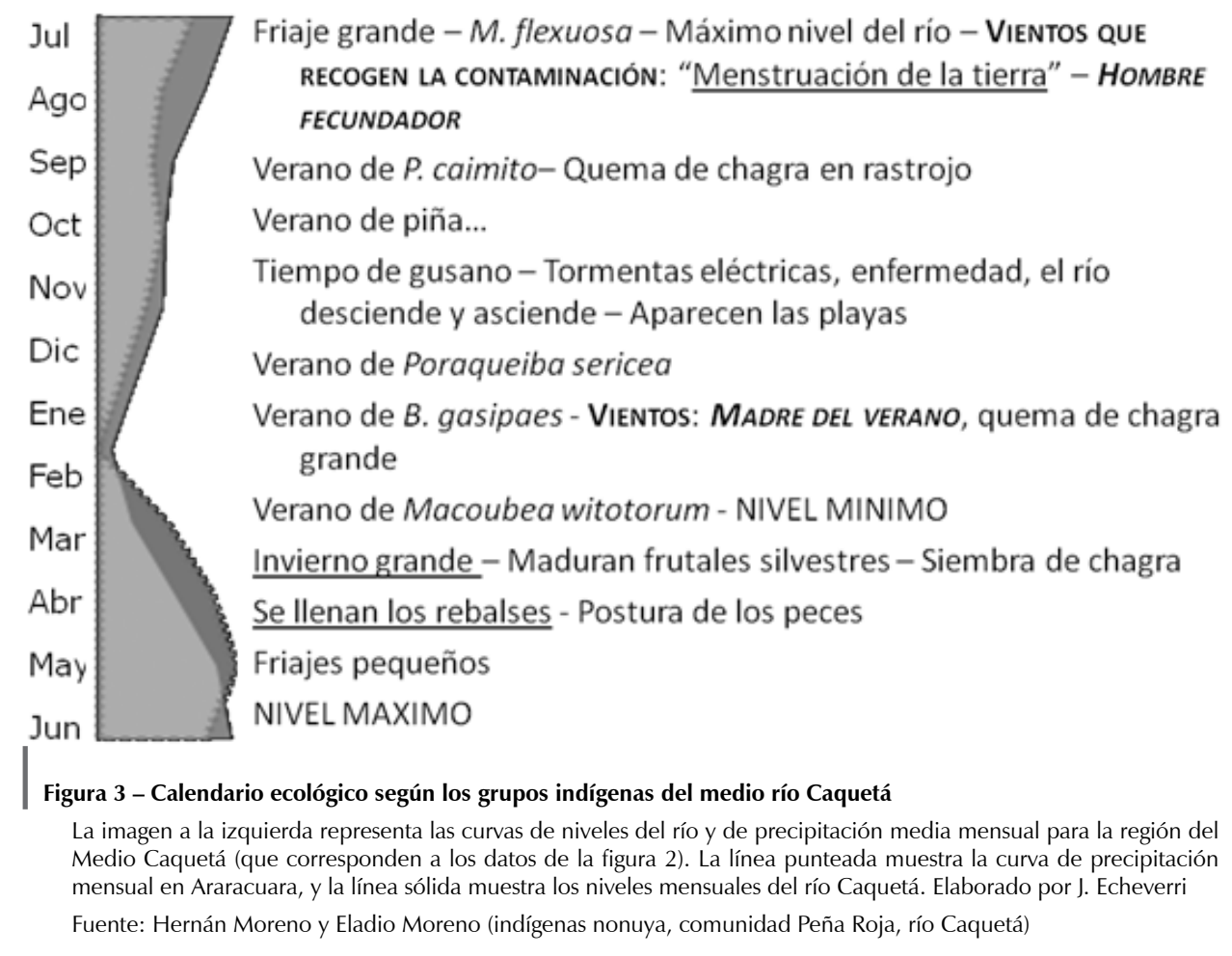

\section{1. El calendario ecológico anual (como debería ser) 5}

El principio del año indígena es la época del tiempo frío, llamada friaje (friagem, en portugués; aru, en la región de Vaupés) en el mes el julio. Normalmente, el friaje ocurre dos o tres veces, siendo la última ocurrencia la más fuerte, coincidiendo con la maduración de las frutas de la palma Mauritia flexuosa (canangucho o aguaje, una palma cuyos frutos son una fuente importante del alimento para la fauna terrestre y aérea). Durante el friaje, que dura tres o cuatro días, hay vientos y llovizna y un descenso notable en la temperatura ambiente (hasta alrededor de $16^{\circ} \mathrm{C}$ ). Los peces mueren en los lagos y en los ríos y las aves no cantan. Para los indígenas, el friaje es la época de la «menstruación» de la tierra. Los vientos de este tiempo breve pero poderoso se conciben como un hombre que viene a impregnar la tierra y fecundarlo todo: las frutas silvestres, la fauna terrestre, los peces, los seres humanos. También trae vida y protección a los humanos, quienes pueden ganar fuerza y habilidades renovadas bañándose temprano durante el tiempo frío. Los vientos del friaje llegan cuando los ríos están en su máximo nivel, y marca el final de la estación de lluvias.

El friaje es seguido por una sucesión de «veranos» (pequeñas estaciones secas), que reciben el nombre de las frutas que maduran en cada uno: verano de Pouteria caimito (caimo), verano de Ananas comosus (piña), y así sucesivamente, separados por periodos de lluvias. Estas breves estaciones secas sirven para tumbar y quemar chacras en rastrojo, y se extienden desde agosto hasta septiembre. 
Estos veranos son seguidos, en octubre y noviembre por el «tiempo de gusano», cuando el bosque se llena de gusanos que destruyen las frutas silvestres. Es una época de tempestades eléctricas seguidas por un calor intenso; el río asciende y desciende, y es una época que favorece la aparición de enfermedades virales.

A medida que el río desciende, viene el verano de Poraqueiba serícea (umarí, un frutal cultivado y silvestre muy apreciado), en diciembre, y el verano grande de Bactris gasipaes (pijuayo, chontaduro), en enero, cuando los ríos alcanzan su nivel más bajo, seguido por el verano de Macoubea witotorum (cocuy, güevas de toro), en febrero. Ésta es la estación seca, cuando se queman las chacras grandes en bosque maduro, con el calor y los vientos fuertes que soplan constantemente desde el este.

Así como los vientos del tiempo de friaje son llamados «Padre impregnador», los vientos del verano grande se conciben como «Madre del verano» que llega a ayudar a preparar las nuevas chacras. En el mes de marzo comienza el tiempo de lluvias, cuando se plantan las chacras nuevas y el río comienza su ascenso constante. Llueve todo abril y mayo, cuando el río inunda los rebalses (llanuras aluviales) e inunda extensas áreas del bosque. En este tiempo maduran muchos frutales silvestres, alimentados por la lluvia, y sus frutos caen sobre las aguas en ascenso. Esto hace que los peces suban y se dispersen a lo largo del bosque inundado para comer el alimento disponible para colocar sus huevos.

El ascenso del río se detiene con la llegada de los friajes que marcan el comienzo de un nuevo año. Las aguas del río comienzan otra vez su descenso, arrastrando con ellas la contaminación de este mundo.

\section{2. Cambios recientes en el ciclo estacional}

Este ciclo anual ideal es afectado por variaciones interanuales recurrentes. Cada número de años ocurren inundaciones excepcionalmente altas (llamadas «conejeras»), y es normal que las estaciones se adelanten o se atrasen a los tiempos previstos. Los indígenas están acostumbrados a cambios en los ritmos estacionales y observan la aparición de marcadores ecológicos y fenológicos que anuncian la sucesión estacional. Lo que es crucial para la vitalidad del sistema, según los indígenas, es la presencia de las dos estaciones de viento durante el friaje y durante el verano grande, la inundación y descenso oportunos de los ríos, acoplado con la sucesión de estaciones lluviosas y secas. Todo esto garantiza la reproducción exitosa de los peces y de la fauna terrestre, la maduración de las frutas y el ciclo ordenado de actividades hortícolas y rituales. En los últimos años, sin embargo, los marcadores ecológicos han venido ocurriendo de forma muy anticipada o retrasada, o desincronizados con la estación que marcaban. Enseguida comento los principales cambios observados.

- Verano (estación seca). Ya no hay más una estación seca claramente marcada con vientos fuertes; hay calor pero no acoplado con los vientos. Hay sucesión de días secos, pero tiene lugar fuera de la época prevista del año: julio-agosto en el régimen Sur, enerofebrero en el régimen Norte. En el 2007, no hubo verano en absoluto en ninguno de los dos regímenes.

- Friaje (estación fría). Está teniendo lugar por adelantado, es menos fuerte o es muy corto. Los vientos del friaje no alcanzan a purificar los árboles, que no llegan a madurar sus frutos. Estos vientos se conciben como colectores de las enfermedades del monte; si no llegan con suficiente fuerza, la contaminación se acumula y afecta la salud natural y humana. Un hombre ticuna de río Amazonas lo expresó de manera sucinta: «los ancianos dicen que al Padre del friaje lo han matado». 
- Precipitación (estación de lluvias). Está desorganizada, no hay estaciones secas o de lluvia claramente demarcadas. «Llueve cuando no debe llover, hace calor cuando no debe hacer calor», afirma un hombre indígena de Araracuara. En el año 2008, cuando se suponía que era el pico de la estación lluviosa (abril), en el río Igaraparana (régimen Norte), tres días de cielos azules y calor intenso fueron seguidos por tormentas y luego por días cubiertos de nubes, amenazando con lluvias que solo fueron llovizna. En el río Amazonas (régimen Sur), mientras tanto, llovía intensamente.

- Niveles del río. Este prominente marcador estacional ha mostrado toda suerte de alteraciones, con diferencias en los dos regímenes. Para la región Norte, en 2005, el río inundó los rebalses, los peces pusieron sus huevos, pero entonces el río descendió abruptamente antes de que los peces estuvieran suficientemente maduros, causando su muerte6. En 2007, vino una inundación alta en el Caquetá y otros ríos del norte, y tuvo lugar una reproducción exitosa de peces, que no había ocurrido desde hace 5 años. En el río Amazonas (régimen Sur), la inundación no ha sido suficiente desde 1999, lo que ha afectado directamente la reproducción de la fauna acuática. El comportamiento anormal de los niveles del río también afecta la reproducción de las tortugas, que requieren la aparición oportuna de las playas para colocar sus huevos. El correcto descenso de los niveles del agua es una condición requerida para la reproducción de tortugas, particularmente de la tortuga gigante Podocnemis expansa (charapa), una especie amenazada. Cuando el río desciende, las tortugas hacen una primera postura en las playas, en noviembre (régimen Norte); normalmente, esta postura es arrastrada por un ascenso final de las aguas. Entonces, las tortugas hacen su postura definitiva a medida que el río sigue descendiendo y entra a la estación seca. En 2005, en el río Caquetá, la segunda postura de las tortugas también fue arrastrada por un ascenso anormal del río.

- Sequía y fuegos. El año 2005 fue un año excepcionalmente seco en la Amazonía meridional y occidental. Fuertes emisiones de humo provenientes de extensos incendios que ocurrieron tan lejos como el occidente de Brasil y el sur de Bolivia, alcanzaron a llegar hasta el río Amazonas —Marengo et al. (2008) muestran que la sequía del 2005 no estuvo ligada a un evento de El Niño, sino a temperaturas superficiales más altas en el Océano Atlántico tropical- En las regiones bajo el régimen Norte, esta sequía no se sintió, sino que causó que el río no se inundara ese año. En Puerto Leguízamo (río Putumayo) y Leticia (río Amazonas), se invirtieron los regímenes de precipitación7.

\section{EFECTOS SOBRE EL MODO DE VIDA INDÍGENA}

Un primer impacto de la alteración de la estacionalidad es sobre la fauna y la flora. Como acabamos de ver, pulsos de inundación del río que no alcanzan a inundar los rebalses o que tiene descensos y ascensos abruptos, afectan directamente la reproducción de la fauna acuática, particularmente de los peces, que constituyen la fuente de proteína más importante para la gente amazónica ${ }^{8}$. Friajes cortos, débiles o fuera de tiempo, junto con

6 Información meteorológica de estación Las Mercedes, río Caquetá $\left(0^{\circ} 32^{\prime} \mathrm{S} 72^{\circ} 10^{\prime} \mathrm{W}\right)$, niveles medios mensuales del río Caquetá para los años 1987-2005 (IDEAM, Colombia, 2008).

7 Datos meteorológicos de las estaciones: Puerto Leguízamo, río Putumayo (00 $\left.{ }^{\circ} 19^{\prime} \mathrm{S}, 74^{\circ} 46^{\prime} \mathrm{W}\right)$, Puerto Arica, río Putumayo $\left(02^{\circ} 08^{\prime} \mathrm{S}, 71^{\circ} 44^{\prime} \mathrm{W}\right)$ y Aeropuerto Vasquez Cobo, río Amazonas $\left(04^{\circ} 12^{\prime} \mathrm{S}, 69^{\circ} 57^{\prime} \mathrm{W}\right.$ ) (Colombia, IDEAM, 2008).

8 Estudios sobre la utilización de recursos en diferentes pueblos de la Amazonía han demostrado claramente que los recursos pesqueros representan la proporción más alta y estable de fuentes de proteína, por encima de la cacería o de la cría de animales; ver, por ejemplo Johnson (2003) para los matsigenka, Cabrera et al. (1999) para los nukak, Descola (1989 [1986]) para los achuar, Van der Hammen (1991) para los yukuna, entre muchos otros. Ver también Dorea (2003). 
la sucesión errática de estaciones secas y lluviosas causan que la floración y maduración de los frutales silvestres ocurra fuera de tiempo o fracase por completo.

Dos cosechas prominentes, que marcan el pico de la estación seca (Bactris gasipaes, la palma de pijuayo o chontaduro) y de la estación fría (Mauritia flexuosa, la palma de aguaje o canangucho) han mostrado un comportamiento anormal en la mayoría de los años recientes. Todo esto, a su vez, tiene un impacto sobre la fauna terrestre, que se alimenta de esas frutas, y sobre los humanos, que se alimentan de ambos.

El incremento de calor afecta sobre todo el trabajo hortícola. Temperaturas más altas, combinadas con los cambios en la precipitación y la estacionalidad, impactan directamente el crecimiento temprano y el éxito de los cultivos. Algunas cosechas necesitan ser replantadas dos o tres veces. La yuca, la cosecha más importante en las chacras indígenas, es más resistente al calor y a la sequía y crece bien en suelos pobres. Esto garantiza una fuente de carbohidratos, pero amenaza la diversidad de cosechas en las huertas.

Los indígenas de esta parte de la Amazonía trabajan por lo menos tres tipos de chacras:

- Chacras tumbadas en bosque maduro sobre suelos de terra firme (suelos ácidos y arcillosos, pobres en nutrientes), que requieren un periodo de sequía más largo y necesitan ser quemados para mejorar el $\mathrm{pH}$ y enriquecer el suelo, son los tipos de chacras con mayor diversidad cultivada.

- Chacras tumbadas en bosques secundarios, que requieren un periodo más corto de sequía, pero son menos productivos y más propensos a la invasión de malezas, son chacras de menor duración y menos diversas.

- Chacras en llanuras aluviales sobre suelos cuaternarios, que se enriquecen con las inundaciones periódicas y no requieren quema, pero sirven solamente para cultivar especies que pueden ser cosechadas antes de que la llanura aluvial sea nuevamente inundada; son las chacras más productivas pero las menos diversas.

Los tres sistemas de cultivo han sido afectados directamente por los cambios recientes en temperatura y estacionalidad, aunque de diferente manera.

Las chacras tumbadas en bosque maduro dependen de la ocurrencia regular de una estación de días secos, calientes y con vientos constantes, que garanticen una quema completa de la parcela. La no ocurrencia de una estación de verano seco en los últimos años ha ocasionado que las parcelas tumbadas se quemen de forma incompleta o no puedan ser quemadas. En el primer caso (quema incompleta), los indígenas tienen que picar y amontonar la madera que no se quemó para hacer hogueras y terminar el proceso de quema, intensificando de esta manera el tiempo de trabajo invertido en el huerto. Este tipo de chacras es trabajado, principalmente, por los grupos indígenas de la Amazonía nororiental colombiana, que tienen abundante acceso a bosque maduro; la gente indígena más cercana a áreas urbanas o de la colonización ya no las hace, pues su acceso está seriamente restringido. Estas chacras de alta diversidad sobre monte firme son importantes no solo como fuente de alimento, sino también para la vida ritual y ceremonial. Así, tanto la diversidad cultivada como la vitalidad del ceremonial en las áreas más tradicionales se ven directamente afectadas por el cambio climático.

Por otra parte, chacras más productivas pero menos diversas sobre suelos aluviales son amenazadas por cambios imprevisibles del nivel del río. Inundaciones súbitas o tempranas pueden destruir cosechas enteras. Este tipo de chacras son hechas sobre todo por gente ribereña, que vive a lo largo de los ríos principales de aguas blancas (Amazonas, Putumayo) y también como alternativa para los grupos tradicionales del río Caquetá. Se utilizan para sembrar cosechas para la venta y para la subsistencia. La inestabilidad de esta forma de 
cultivo, debido a patrones cambiantes e impredecibles de los niveles del río, a largo plazo, afecta la economía de subsistencia y de mercado de grupos menos tradicionales, y también las formas alternativas de horticultura de grupos tradicionales.

Las chacras sobre bosque secundario - menos diversas que las de bosque maduro y menos productivas que las de suelos aluviales - aparecen entonces como la alternativa más confiable. Ha llegado a ser habitual para aquellos grupos con territorios restringidos y es una alternativa para la gente ribereña como para los grupos tradicionales de tierra firme. Estas chacras demandan menos trabajo y no requieren de una estación seca prolongada para quemarse con éxito. La tumba repetida del bosque secundario, sin embargo, conduce a la degradación de los suelos y a la creciente invasión de malezas.

Otro efecto de los cambios climáticos recientes ha ocurrido sobre la salud. La mayoría de las personas entrevistadas ha subrayado la ocurrencia creciente de enfermedades respiratorias e intestinales, no solo más fuertes, sino también de tipos no conocidos antes. Las enfermedades transmitidas por vectores, como malaria y dengue, al parecer no han tenido incrementos, pero el carácter cambiante de la fluctuación de los ríos puede crear condiciones favorables para la reproducción de vectores de maneras aún imprevisibles.

Los indígenas reconocen la recurrencia estacional de enfermedades virales y recurren a prácticas preventivas de naturaleza material como espiritual. La aparición y desaparición de enfermedades recurrentes (gripes, diarreas) está muy relacionada con los pulsos de inundación, los regímenes de precipitación y los cambios de temperatura. La enfermedad, en la visión de los grupos del Caquetá-Putumayo, está en los árboles y en el agua; la inundación y los vientos del friaje sirven para colectar toda esa enfermedad del mundo y drenarla hacia el mar cuando los ríos descienden. Cuando la inundación no es suficientemente alta o el friaje no es fuerte, la enfermedad se acumula en la atmósfera y en las aguas, haciendo que los trastornos respiratorios e intestinales se prolonguen y aparezcan otros nuevos. Especialistas rituales de la región del medio Caquetá (régimen Norte) expresan claramente que las prácticas y prevenciones rituales que han servido para prevenir y controlar los efectos adversos de cada estación, cada vez están teniendo menos efecto, debido al desorden de las estaciones. El calor también afecta la salud humana. Las altas temperaturas en áreas abiertas, como las chacras en producción, causan fatiga y dolores de cabeza, y la temperatura más alta incrementa, en la visión indígena, la circulación de la enfermedad en el mundo.

Otros efectos sobre la salud humana — no evidentes aún, pero predecibles - tienen que ver con la potencial disminución de las fuentes de alimento. Los peces constituyen la principal fuente de proteína para las poblaciones humanas, y son uno de los recursos más directamente afectados por la alteración en el régimen de estacionalidad. Los impactos sobre el sistema de chacras afecta tanto la disponibilidad de carbohidratos (pequeñas chacras en bosque secundario no producen la cantidad de yuca para una familia) y la diversidad de otros tubérculos y frutos cultivados (las chacras son menos diversas), que enriquecen y complementan la dieta. Fuentes alternativas de proteína (fauna terrestre, avifauna, insectos) y de vitaminas y minerales (frutas silvestres) son también directamente afectadas.

\section{1. Género, edad y situación social}

Aunque la mayor parte de estos cambios tiene efectos para todas las categorías de género y edad, algunos grupos son más vulnerables a impactos actuales y potenciales. Además, dependiendo de las condiciones ecológicas (régimen Norte, régimen Sur, piedemonte, selva oriental) y sociocultural (acceso a territorio, recursividad cultural, contacto) se configuran escenarios de impacto diferenciales. 
Los infantes y niños son el grupo más vulnerable a enfermedades virales. También la disminución en la disponibilidad de proteínas y las fallas en las cosechas afectan directamente el crecimiento y desarrollo sano de los segmentos más jóvenes de la sociedad.

Los especialistas rituales, quienes dependen en gran medida de la producción de comida cultivada - abundante, diversa y de amplio acceso a recursos de cacería—, son impactados por la dificultad creciente de establecer chacras sobre monte firme, que son requeridas para llevar a cabo las actividades rituales. Enfermedades nuevas y más fuertes también menoscaban sus capacidades de curación y prevención.

Las mujeres en edad adulta reciben la mayor carga de impacto con estos cambios, al ser las mujeres quienes tradicionalmente están a cargo del trabajo hortícola. El trabajo en campos abiertos bajo temperaturas crecientes, la pérdida de cosechas, la necesidad de replantar y los rendimientos menores de las cosechas afectan su salud y autoestima. Las chacras en bosque secundario o en bosque maduro que no queman lo suficiente incrementan el tiempo requerido para erradicar malezas o terminar manualmente el proceso de quemado. La mala salud y la desnutrición de los niños afectan directamente el trabajo y las responsabilidades de las mujeres. Hombres jóvenes que son responsables por la adquisición de proteínas y la apertura de nuevas chacras, son afectados por la disminución en la disponibilidad de los recursos de pesca. El esfuerzo para conseguir cacería terrestre y pescado aumenta para ellos, y los criterios para decidir los tiempos correctos para tumbar y quemar chacras se vuelven más inciertos.

Los grupos indígenas con mayor autonomía territorial que derivan su sustento (sobre todo) de recursos del bosque y del agua, y mantienen una vida ritual activa, son los más fuertemente afectados en su subsistencia. Estos grupos valoran, en gran medida, las chacras en bosque maduro plantadas con gran variedad de especies, dependen fuertemente de la pesca y la cacería como fuente de proteínas, y cuidan su salud con sus propios medios y conocimientos. Su subsistencia se basa en su capacidad para interpretar los ciclos naturales regulares y de actuar en consecuencia. Ciertamente, estos grupos tienen contacto con la sociedad nacional, están incorporados en alguna medida a la economía de mercado y tienen acceso a los servicios de salud y educación públicas. Un porcentaje grande de su subsistencia depende de su conocimiento, uso y manejo de los recursos del bosque y del agua. Estos son los grupos más conscientes de estos cambios y también los más vulnerables a sus efectos. En esta categoría están los grupos indígenas del área de Vaupés (familias lingüísticas Tukano oriental, Maku-Puinave y Arawak), de la región del Caquetá-Putumayo (familias lingüísticas Witoto, Bora-Miraña y Andoke), de la región de Guainia (familias lingüísticas Maku-Puinave y Arawak) y de algunos grupos del Trapecio amazónico (familias lingüísticas Tikuna, Peba-Yagua y Tupi).

Un escenario distinto ocurre con los grupos indígenas con autonomía territorial restringida y poco o ningún acceso al bosque maduro, que dependen de la horticultura en bosque secundario, cosechas para la venta sobre suelos aluviales, pesca comercial, trabajo asalariado, turismo y venta de las artesanías para su subsistencia. Ellos se ven afectados por los cambios mencionados arriba, en la medida que utilizan los recursos del río y del bosque, y son igualmente afectados por las enfermedades y los incrementos de temperatura. Estos grupos indígenas están conscientes del calendario estacional (o restringido a esos aspectos que afectan directamente sus actividades), su conocimiento tradicional es más limitado y los especialistas rituales, por lo general, tienen un papel menos central en la sociedad. Su mayor acceso al mercado y a los servicios de salud y educación públicas les ofrece recursos para amortiguar los impactos sobre su subsistencia. Son menos conscientes y menos vulnerables que los grupos más tradicionales, pero el efecto combinado del cambio climático tiende a acelerar la incorporación al trabajo asalariado, la integración al mercado, la migración 
hacia áreas urbanas y la pauperización. En esta categoría están los grupos indígenas en las zonas de colonización del piedemonte andino y a lo largo del río de Guaviare, y los grupos indígenas de las otras áreas que viven en la vecindad de las urbes.

\section{2. Interpretación indígena del cambio climático}

La interpretación nativa de las alteraciones climáticas en curso, para el caso de los grupos del Caquetá-Putumayo, tiende a basarse sobre la responsabilidad humana. Los indígenas ciertamente han escuchado, en las noticias y a través de visitantes, sobre el cambio global del clima, pero principalmente se refieren a ellos mismos como causa de los cambios que afectan su subsistencia y bienestar. Puede ser verdad que «los blancos» han contaminado la tierra con sus fábricas y sus bombas, pero también es verdad que los indígenas, dicen ellos mismos, tienen una parte de la responsabilidad por no vivir y manejar la vida como es debido. Según la Historia mítica —ellos narran—, anteriormente los seres humanos manejaban el tiempo y el territorio según las fases del ciclo anual. Al principio del año —durante la época de friaje- los ancianos hablaban del principio de la vida y del crecimiento y rechazaban todo el mal. Esto se hacía por medio de diálogos y bailes rituales. En la siguiente fase, evaluaban la estación anterior antes de preparar la venidera, y así se continuaba hasta que el ciclo completo terminaba - siempre con actividades rituales que requerían productos hortícolas abundantes y diversos-. Cuando tumbaban el monte, llamaban a la Madre del Verano. Después de quemar llamaban al Abuelo de la Lluvia, y en su diálogo hablaban sobre los procesos de gestación para hacer que las semillas brotaran. Este comportamiento, afirman, contribuía a que la sucesión de las estaciones fuera regular y que las cosechas fueran abundantes. «Hablando de esta manera, la naturaleza se mantenía saludable. Si las plantas están bien, entonces los niños y las mujeres están bien y saludables. Hoy en día esto ya no se practica, aunque el conocimiento existe», sentencian con preocupación. El desorden en la naturaleza es reflejo del desorden en la sociedad.

Es lo social, no lo natural, lo que está en el centro de la interpretación indígena amazónica de la situación actual —incluido el clima- La sociedad humana está afectada no solo por la inestabilidad de las condiciones naturales, sino también por todos los otros cambios y transformaciones generados en su modo de vida, por el contacto con la economía de mercado, la educación escolarizada, los medio masivos de comunicación y el contacto con toda la clase de agentes externos. Estas nuevas condiciones han erosionado precisamente el manejo de la vida a través del diálogo y las prácticas rituales. De nuevo, la culpa, en su visión, no se pone en la gente blanca que los ha conquistado y asimilado cada vez más en una sociedad global, sino en la falta de capacidad de los indígenas para manejar y resistir a estos cambios con maneras innovadoras. Así, el cambio climático y el cambio social están interrelacionados y se ubican en una perspectiva ética que incorpora tanto las maneras tradicionales de hablar y relacionarse con las entidades naturales, y las nuevas maneras de lidiar con la sociedad envolvente.

\section{ESTRATEGIAS DE ADAPTACIÓN Y SUPERVIVENCIA}

Los indígenas amazónicos están acostumbrados a cambios y variaciones en el clima y la estacionalidad, tanto cambios anuales recurrentes como variaciones interanuales. Una de las principales características del modo de vida amazónico, común a grupos indígenas

9 Esta forma de diálogo, que interpela los elementos naturales (el verano, las lluvias, el fuego, el viento) en términos de relaciones sociales, hace parte del diálogo ceremonial de tabaco y coca de los grupos del interfluvio CaquetáPutumayo o «Gente de centro» (Echeverri, 1997). 
y mestizos, es la «pluriactividad», es decir, el conocimiento de un conjunto variado de habilidades que permiten que la gente haga muchas actividades diversas en ambientes ecológicos diversos. Los indígenas son pescadores, horticultores, cazadores, conocen el río y el bosque, y pueden también aprender nuevas técnicas de la gente blanca y utilizarla en su beneficio. Esta capacidad pluriactiva es un gran recurso cultural, común a todos los grupos, que les permite adaptarse y hacer frente a múltiples cambios en su entorno. Como afirmó un hombre cocama del río Amazonas, «tenemos que aprender a hacer de todo para sobrevivir». Sin embargo, los extremos climáticos ponen a prueba estas capacidades y los indígenas están ahora en un proceso de búsqueda para solucionar amenazas inminentes a su supervivencia, que son no solamente climáticas sino también sociales.

\section{1. Horticultura}

La horticultura es uno de los sistemas de subsistencia fundamentales que ahora está en mayor riesgo, particularmente las chacras abiertas en bosque maduro. La carencia de una estación seca claramente marcada ha implicado cambios: "ya no esperamos el verano», afirma un hombre del río Caquetá. Se exploran varias alternativas:

- Tumbar monte y quemar tan pronto haya algunos días secos.

- Si esto no sucede, construir hogueras e ir quemando la chacra poco a poco.

- Preferir abrir chacras en bosques secundarios que requieren periodos secos menos prolongados.

- Hacer chacras sin quemar sobre suelos aluviales, con el riesgo de perder las cosechas si el río crece antes que las plantas estén listas.

Las dos últimas opciones conducen a cultivos con menor diversidad de especies, y sobre suelos más aptos para las variedades comestibles de yuca (que crecen más rápidamente que las amargas). Para los grupos indígenas más tradicionales de la Amazonía nororiental colombiana, estas adaptaciones tienen impacto sobre la diversidad de especies cultivadas y sobre la vida ceremonial. Para las poblaciones ribereñas y los grupos indígenas con menor autonomía territorial, estos cambios ya han estado teniendo lugar hace algunos años. Parece entonces que el sistema de tumba y quema de chacras sobre monte maduro, como ha sido documentado por etnógrafos, pronto será un hecho de la Historia, en la medida en que muchos grupos tenderán a adoptar sistemas menos diversos en especies y también menos dependientes de condiciones estacionales marcadas.

Otro cambio en las prácticas hortícolas es el de prolongar la vida de los campos de cultivo. Las chacras tradicionales indígenas no duran más allá de algunos años y después se abandonan para convertirse en rastrojo. La gente amazónica no acostumbra hacer prácticas de enriquecimiento del suelo. La idea de hacer prácticas de abono y mantenimiento del suelo no ha surgido de los indígenas mismos sino que ha sido promovida por el Instituto Sinchi, una institución gubernamental, en el Trapecio amazónico y en el río de Igaraparana (región de Caqueta-Putumayo). Ellos abogan porque no se hagan quemas del bosque y en su defecto, se plante en campos ya existentes, manteniendo la productividad y agregando fertilizantes naturales (cenizas, madera podrida, etc.). Este sistema está apenas siendo introducido y sus resultados todavía esperan por verse. Esto conduce a una dedicación mucho más intensa a la horticultura, que compite con el tiempo disponible para otras actividades de subsistencia, menoscabando así la pluriactividad. 


\section{2. Más allá del cambio climático}

Hoy la preocupación más grande de los indígenas, más que las dificultades con la productividad de las chacras o la pesca, es su situación social. La contaminación que destruye los elementos de la vida no es solo la natural, sino también el desorden social, que es a su vez exacerbado por el cambio climático. La producción no está solo afectada por los cambios en la estacionalidad, sino también por los efectos negativos de la explotación inadecuada de los recursos, la destrucción del bosque, la minería aurífera, el tráfico ilegal de coca; factores que incrementan esa otra contaminación.

Es verdad que muchas de esas acciones destructivas se originan en procesos y agentes externos, pero lo que más preocupa a los indígenas es cómo se ven implicados y transformados por ellas. Uno de los factores que más incide en esto es la necesidad del dinero. El dinero se necesita para que los jóvenes asistan a la educación nacional, una preocupación dominante de los indígenas. Los hombres jóvenes emigran fuera de la región, para trabajar en cultivos de coca o en extracción de madera, buscando dinero para sostener una familia. Los hombres explotan recursos naturales para conseguir el dinero para satisfacer las demandas de mujeres y de niños. «Estamos enceguecidos por el dinero, los productos del bosque ahora se han vuelto negocio»; dice un hombre uitoto de la región del río Amazonas. El cambio climático afecta la producción hortícola y la oferta de proteínas disminuye incitando a la gente a emigrar. «Antes, eran individuos que emigraban, ahora son familias enteras que salen de los Resguardos»; declaró un hombre nonuya del río de Caquetá.

Los efectos del cambio climático global han comenzado a hacerse sentir en este ambiente social también cambiante y amenazador. En una reunión reciente (2008) de los pueblos muinane y nonuya, en el río Caquetá, los ancianos estuvieron evaluando estos asuntos. Ellos trataron sobre dos tendencias interrelacionadas que afectan directamente sus vidas: problemas con la producción (horticultura, pesca, cacería) y problemas sociales (guerrilla, comercio ilícito, explotación de recursos naturales, migración, problemas de salud). Su evaluación de causas y sus propuestas de acción nos dan un cuadro claro de los dilemas actuales de la gente indígena:

- Como ancianos, no están practicando el control ritual de la naturaleza y de la sociedad que fue establecido por el Creador para atenuar y para invertir los efectos que ahora atestiguan. El desorden natural es un reflejo del desorden social. No es el trabajo de una sola persona, se requiere la coordinación entre especialistas rituales de diversas tribus.

- Las autoridades indígenas formales (gobernadores, cabildos electos y los líderes políticos indígenas) no tienen el conocimiento ni la capacidad adecuados para ocuparse de estas situaciones, porque están dominados por las agendas y el modo de pensar de los blancos. Le compete a los ancianos y a los jefes de familia reasumir sus responsabilidades morales.

- Las agendas y las preocupaciones de la gente blanca están dominando, cada vez más, los tiempos y los espacios que se deben dedicar al diálogo y a las prácticas rituales y a la educación tradicional de nuevas generaciones. Es necesario hacer esfuerzos para controlar y «enfriar» estas interferencias dañinas.

- El peligro más grande viene «de la palabra caliente y venenosa» que viene del subsuelo, donde debe mantenerse guardada. La gente blanca la ha liberado extrayendo petróleo, y ahora llega hasta los indígenas en forma de dinero, armas, lujos, alcohol, medios masivos y enfermedades - y también en una atmósfera cambiante-.

«Si todos estos cambios son el resultado de un desorden planetario, ¿qué podemos nosotros, un pequeño grupo de gente, lograr?»; se preguntan y plantean una responsabilidad compartida. Necesitan la ayuda y la interlocución exteriores para dar a conocer su propio 
diagnóstico y ofertas, y para demostrar sus sistemas de manejo natural. Pero esta relación con las instituciones exteriores tiene que tomarse de una nueva manera, no aceptando y recibiendo propuestas elaboradas fuera, como ha sido la regla, sino buscando ayuda y apoyo para sus propias agendas y sus propias preocupaciones.

\section{Referencias citadas}

BUNYARD, P., 2008 - Why Climate is Dependent on Biodiversity. In: Fronteras en la globalización: localidad, biodiversidad y comercio en la Amazonía (C. Zárate \& C. Ahumada, eds.): 21-42; Bogotá: Fundación Konrad Adenauer, Pontificia Universidad Javeriana.

CABRERA, G., FRANKY, C. E. \& MAHECHA, D., 1999 - Los Nukak: Nómadas de la Amazonía colombiana, 424 pp.; Bogotá: Universidad Nacional de Colombia, Fundación GaiaAmazonas.

DESCOLA, P., 1989 [1986] - La selva culta: simbolismo y praxis en la ecología de los Achuar, 468 рp.; Quito: Abya-Yala.

DOREA, J. G., 2003 - Fish are central in the diet of Amazonían riparians: should we worry about their mercury concentrations? Environmental Research, 92 (3): 232-244.

INTERGOVERNMENTAL PANEL ON CLIMATE CHANGE (IPCC), 2007 - Climate Change 2007: Synthesis Report. Intergovernmental Panel on Climate Change Web Site $<$ http://www.ipcc.ch/pdf/assessment-report/ar4/syr/ar4_syr.pdf $>$.

JOHNSON, A., 2003 - Families of the Forest: The Matsigenka Indians of the Peruvian Amazon; Berkeley/Los Angeles/London: University of California Press.

MARENGO, J. A., NOBRE, C. A., TOMASELLA, J., CARDOSO, M. F. \& OYAMA, M. D., 2008 - Hydro-climatic and ecological behaviour of the drought of Amazonia in 2005. Philosophical Transactions of the Royal Society, B 363: 1773-1778. Published online <http://journals.royalsociety.org/content/238x818l0815588k/fulltext.pdf $>$.

SALICK, J. \& BYG, A., 2007 - Indigenous Peoples and Climate Change; Oxford: Tyndall Center for Climate Change Research. Published online: $<$ http://www.tyndall.ac.uk, publications/Indigenouspeoples.pdif $>$.

VAN DER HAMMEN, M. C., 1992 - El manejo del mundo: Naturaleza y sociedad entre los Yucuna de la Amazonía colombiana, 376 pp.; Bogotá: Tropenbos. 